\title{
Importância das características na precificação de veículos nacionais
}

\author{
Camilo Brandão de Resende ITA \\ Rodrigo Arnaldo Scarpel ITA
}

\section{RESUMO}

O mercado de bens de consumo é constituído por produtos heterogêneos que podem ser vistos como uma união de atributos ou características. Quantificar o valor dessas características pode auxiliar na criação de uma regra de precificação que permita, por exemplo, precificar um produto não existente, auxiliando na sua previsão de demanda. 0 objetivo deste trabalho é utilizar a teoria do preço hedônico para determinar empiricamente o valor e a importância relativa das características na precificação de veículos nacionais brasileiros. 0 modelo de previsão foi obtido utilizando-se uma função hedônica linear, cujos coeficientes foram estimados através de mínimos quadrados ordinários. Pode-se concluir que a potência é o fator de maior impacto no preço de um veículo, seguido pelo grau de luxo e marca.

PALAVRAS-CHAVE

Precificação, regressão hedônica, mercado automobilístico.

\section{Importance of characteristics in pricing national vehicles}

\begin{abstract}
The market of consumer goods is consisted of heterogeneous products which can be viewed as a union of attributes or characteristics. Quantifying the value of these characteristics can help in the creation of a pricing rule, allowing, for example, pricing a product which is not available, and helping in the estimates of its demand. The purpose of this work is to use the theory of hedonic price to determine empirically the value of characteristics, as well as their relative importance, in the price of Brazilian vehicles. The model was obtained using data regarding 150 national vehicles and a linear hedonic function, which coefficients were estimated by ordinary least squares. It can be concluded that the engine power is the factor which has the greatest impact on a vehicle price, followed by the luxury level and the brand.
\end{abstract}

\section{KEY WORDS}

Pricing, hedonic regression, auto market. 


\section{INTRODUÇÃO}

O mercado de bens de consumo é, sabidamente, constituído por produtos heterogêneos. Esses produtos podem ser vistos, em geral, como uma união de atributos ou características. O mercado automobilístico é um exemplo claro da heterogeneidade de produtos, uma vez que as montadoras de automóveis procuram aumentar suas participações no mercado, assim como lucros, produzindo veículos com diferentes características (conforto, potência, tamanho etc.).

Uma das necessidades atuais dos produtores de bens de consumo é quantificar a "real modificação de preço" de um produto ao adquirir determinada característica (BRACHINGER, 2002). Somente com essa quantificação é possível estimar o preço de mercado de um produto antes de seu lançamento, assim como tomar uma decisão quanto a qual característica deve ser adicionada ao produto a fim de aumentar suas vendas e/ou margem de lucro. Linz (2004) afirma que essa quantificação pode ser obtida através de um ajuste hedônico, que utiliza análise de regressão para estabelecer uma relação matemática entre a qualidade de um item - que é determinada por suas características - e o preço pelo qual ele é vendido.

O objetivo deste trabalho é utilizar a teoria do preço hedônico para determinar empiricamente o valor e a importância relativa das características no preço dos veículos nacionais brasileiros, possibilitando previsões de preços de veículos que não existem no mercado.

O modelo foi obtido utilizando-se uma regressão hedônica linear e teve três etapas distintas: (1) a amostra obtida foi dividida em duas partes, sendo três quartos da amostra destinados à formulação do modelo e um quarto ao teste do modelo; (2) os pesos das características foram estimados utilizando a amostra de formulação; (3) o modelo obtido foi testado comparando-se os preços estimados para a amostra de teste com os preços efetivamente praticados pelo mercado.

Após a obtenção do modelo, simulou-se sua utilização, através da comparação entre o preço previsto e o preço real de um veículo de passeio nacional que foi lançado após a coleta dos dados utilizados no presente trabalho.

\section{TEORIA DO PREÇO HEDÔNICO}

Os produtos presentes no mercado sofrem constantes modificações. Então, não podemos mais comparar diretamente os produtos disponíveis atualmente no mercado com produtos que existiam antes. Para comparações de preço, esses devem ser qualitativamente ajustados.

$\mathrm{O}$ ajustamento qualitativo é comumente considerado um dos problemas mais complicados na estatística dos preços.
Técnicas como "linking" ou "overlap pricing" estão sendo atacadas depois da publicação do "Boskin-Report" (BOSKIN et al., 1996) por serem, sob algumas condições, tendenciosas. Os métodos hedônicos foram então recomendados como uma alternativa razoável (BRACHINGER, 2002).

O ponto inicial de todo índice de preço hedônico é a hipótese hedônica. O centro dessa hipótese é que cada bem é caracterizado pela união de todas as suas características. Dado qualquer bem, façamos essa união ser ordenada e denotada pelo vetor $\mathrm{x}=\left(\mathrm{x}_{1}, \ldots, \mathrm{x}_{\mathrm{K}}\right)^{\prime}$. Assumiremos que a preferência por qualquer bem é determinada somente por seu vetor de características correspondente.

Além disso, também é assumido que, para qualquer bem, existe um relacionamento funcional $\mathrm{f}(\mathrm{x})$ entre seu preço e o vetor de características $\mathrm{x}$, i. e.

$$
p=f(x) .
$$

Essa função especifica a relação hedônica ou regressão hedônica típica para o bem. Funções de preço hedônico podem ser vistas como resumos empíricos da relação entre os preços e as características de bens vendidos em mercados contendo produtos diferenciados (PAKES, 2003).

Baseado no relacionamento funcional (1), o importante conceito de preços hedônicos - ou implícitos - pode ser introduzido. Esses preços são definidos como as derivadas parciais da função hedônica (1), i.e.

$$
\frac{\partial p}{\partial x_{K}}(x)=\frac{\partial f}{\partial x_{K}}(x) \quad(\mathrm{k}=1, \ldots . ., \mathrm{K}) .
$$

O preço hedônico $\partial \mathrm{f} / \partial \mathrm{x}_{\mathrm{k}}(\mathrm{x})$ indica quanto o preço $\mathrm{p}$ de um bem varia se esse bem for, ceteris paribus, dotado com uma unidade adicional da característica $\mathrm{x}_{\mathrm{k}}$.

Para aplicações práticas da relação hedônica (1) em estatística de preços, os problemas principais são a determinação do vetor de características de um bem e a especificação da função hedônica.

Segundo Neto (2002), a análise empírica baseada na abordagem hedônica deve responder a duas questões, inicialmente propostas por Griliches (1961): a) Quais são as características relevantes? b) Qual é a forma de relacionamento entre os preços e as características? Portanto, o presente trabalho procurará responder a estes questionamentos aplicados ao mercado automobilístico brasileiro.

\subsection{Origem da teoria do preço hedônico}

Segundo Nervole (1995), a análise do preço hedônico se originou na economia agrícola, quando F. V. Waugh (1929) publicou seu pioneiro estudo sobre fatores que influenciam 
os preços dos vegetais. Ainda segundo Nervole (1995), Waugh fez uma regressão dos preços por lote de aspargos em Boston (maio-junho, 1927) sob três diferentes dimensões de qualidade: avaliação da cor, tamanho da haste e uniformidade dos brotos. Seu objetivo era determinar as valorizações relativas que os consumidores davam a essas características, as quais considerava como informações úteis para os produtores de aspargos.

Colwell e Dilmore (1999) afirmam que Haas (1922) já utilizou o conceito de "hedônico", e fez um modelo simples de preço hedônico para fazendas, considerando a distância para o centro da cidade e o tamanho da cidade como duas importantes variáveis.

A fundamentação teórica do modelo de preço hedônico foi chamada de teoria do preço hedônico, possuindo fundamentalmente dois alicerces:

Inicialmente, o acadêmico americano Lancaster (1966) divulgou uma nova teoria do consumidor. A teoria é uma expansão da teoria econômica clássica, também conhecida como teoria das preferências de Lancaster. A partir da heterogeneidade dos produtos, Lancaster analisou "elementos básicos" que formam o produto, e argumentou que a demanda por um produto não dependia do produto propriamente dito, mas sim de suas características. Produtos heterogêneos (como automóveis) possuem uma série de características integradas, e são vendidos como uma reunião de características inerentes. Famílias compram esses bens, utilizando-os como uma espécie de investimento e os transformam em utilidade. O nível de utilidade depende da quantidade de diferentes características. É muito difícil analisar o mercado de bens de consumo com o modelo econômico tradicional, porque não podemos considerar apenas um preço total. Por isso adotamos uma série de preços (preços hedônicos) para expressar as correspondentes características dos produtos. Portanto, o preço de um produto é formado por preços hedônicos, com cada característica do produto possuindo seu próprio preço implícito e todos os preços hedônicos formam uma estrutura de preço.

Em seguida, o economista americano Rosen (1974) desenvolveu um modelo de equilíbrio de oferta e demanda baseado nas características de produtos. Utilizando a condição de um mercado perfeitamente competitivo, maximizando a utilidade dos consumidores e o lucro dos produtores como meta, Rosen analisou teoricamente o equilíbrio a curto e longo prazo do mercado de produtos heterogêneos. $\mathrm{O}$ trabalho de Rosen estabeleceu a fundamentação de um modelo para a teoria do preço hedônico, baseada em métodos econométricos que podem ser utilizados para estimar a função de preço hedônico, obter os preços implícitos das características dos produtos e analisar a demanda pelas características dos produtos.
Modelos hedônicos já foram utilizados diversas vezes no mercado automobilístico. Segundo Goodman (1998), o primeiro modelo de preço hedônico para o mercado automobilístico foi desenvolvido por Andrew T. Court (1939), analista da indústria automobilística americana. Ele adotou o termo "hedônico" (tendência para agir de maneira a evitar o que é desagradável e a atingir o que é agradável), considerando o preço dos automóveis como uma função de suas diferentes características, e realizou a análise de preço hedônico de bens heterogêneos. Sua proposta final era estruturar um índice de preços para a indústria automobilística. Mais tarde, Griliches (1961) também rodou regressões similares com o propósito de descobrir as preferências dos consumidores em relação a vários opcionais nos automóveis que compravam.

Posteriormente, modelos hedônicos foram utilizados buscando diversos objetivos. Por exemplo, Fisher et al. (1962) usaram regressões hedônicas para avaliar mudanças nos custos de automóveis entre os anos de 1949 e 1961. Cowling e Cubbin (1971), Boyle e Hogarty (1975) e Cubbin (1975) usaram regressões hedônicas para estudar o comportamento competitivo e de precificação nos mercados automobilísticos norte-americano e britânico. Agarwal e Ratchford (1980) e Argua et al. (1994) utilizaram de regressões hedônicas para estimação de funções de demanda para características de veículos. Mais recentemente, Reis e Silva (2006) utilizaram regressões hedônicas para estudar os efeitos de mudanças na qualidade sobre os preços de veículos novos no mercado português.

\subsection{Formato das funções utilizadas nas regressões hedônicas}

Em aproximações hedônicas para problemas de índices de preço, quatro diferentes formas de funções foram empregadas no passado (NETO, 2002): linear, exponencial, dupla logarítmica e logarítmica.

A aproximação mais simples é a linear, que é dada por

$$
p=\beta_{0}+\sum_{k=1}^{K} \beta_{k} x_{k}
$$

com os preços hedônicos

$$
\frac{\partial p}{\partial x_{K}}(x)=\beta_{k} .
$$

O coeficiente da regressão $\boldsymbol{\beta}_{k}(\mathrm{k}=1, \ldots \ldots, \mathrm{K})$ indica a variação marginal do preço com respeito a uma mudança da $\mathrm{k}$-ésima característica $\mathrm{x}_{k}$ de um bem.

Outra aproximação é a exponencial, que é caracterizada por

$$
p=\beta_{0} \prod_{k=1}^{K} \exp \left(\beta_{k} x_{k}\right)
$$


ou

$$
\ln p=\ln \beta_{0}+\sum_{k=1}^{K} \beta_{k} x_{k}
$$

com os preços hedônicos

$$
\frac{\partial p}{\partial x_{K}}(x)=\beta_{k} p
$$

Obviamente, nessa aproximação, os coeficientes da regressão podem ser interpretados como taxas de crescimento. O coeficiente $\boldsymbol{\beta}_{k}(\mathrm{k}=1, \ldots \ldots, \mathrm{K})$ indica a taxa na qual o preço aumenta a um certo nível, dado o vetor característico $\mathrm{x}$.

Uma terceira aproximação é a função dupla logarítmica, que é descrita por

$$
p=\beta_{0} \prod_{k=1}^{K} x_{k}^{\beta_{k}}
$$

ou

$$
\ln p=\ln \beta_{0}+\sum_{k=1}^{K} \beta_{k} \ln x_{k}
$$

com os preços hedônicos

$$
\frac{\partial p}{\partial x_{K}}(x)=\frac{\beta_{k}}{x_{k}} p .
$$

Nessa aproximação, os coeficientes da regressão podem ser interpretados como elasticidades parciais. O coeficiente $\boldsymbol{\beta}_{k}(\mathrm{k}=1, \ldots, \mathrm{K})$ indica em qual porcentagem o preço $\mathrm{p}$ aumenta em um certo nível se a k-ésima característica $\mathrm{x}_{k}$ aumenta em um por cento.

Uma quarta forma de aproximação é a forma logarítmica dada por

$$
p=\beta_{0}+\sum_{k=1}^{K} \beta_{k} \ln x_{k}
$$

com os preços hedônicos

$$
\frac{\partial p}{\partial x_{K}}(x)=\frac{\beta_{k}}{x_{k}} .
$$

De acordo com Neto (2002), a teoria econômica ainda não desenvolveu um critério de escolha para a forma funcional, assim sendo, a maioria dos pesquisadores vê a escolha como uma questão empírica para ser decidida pela melhor adequação aos dados.

\section{ANÁLISE DOS FATORES DETERMINANTES NA PRECIFICAÇÃO DE VEÍCULOS NACIONAIS}

\subsection{Dados utilizados}

A fim de se construir um modelo de previsão de preços de veículos nacionais, foram obtidas as informações apre- sentadas na Tabela 1 a respeito de 150 modelos de veículos produzidos no Brasil. Os preços sugeridos pelas montadoras para veículos novos (sem frete) foram colhidos durante doze meses, utilizando-se as edições de janeiro a dezembro de 2005 da Revista Quatro Rodas. No modelo construído, foi utilizada a média aritmética dos preços pesquisados durante os 12 meses. As demais informações (altura, comprimento etc.) foram obtidas nos web-sites oficiais das montadoras. Os dados utilizados são mostrados no Apêndice A.

Tabela 1: Informações obtidas a respeito de 150 veículos nacionais.

\begin{tabular}{c}
\hline MODELO \\
Marca \\
Tipo de Combustível (Álcool, gasolina ou flex) \\
Potência do motor (cv) \\
Tamanho do porta-malas (I) \\
Comprimento (cm) \\
Altura (cm) \\
Quantidade de portas \\
Tipo de câmbio (mecânico ou automático) \\
Preço sugerido pela montadora (RȘ)
\end{tabular}

\subsection{Características consideradas}

No presente trabalho, as características consideradas são definidas pelas variáveis binárias apresentadas na Tabela 2 .

As faixas de potência foram determinadas empiricamente e o critério utilizado para a escolha das faixas de comprimento foi a segmentação visual baseada na Figura 1.

\subsection{Estimação dos parâmetros}

Pode-se, utilizando a teoria do preço hedônico, escrever uma relação funcional entre o preço $p_{i}$ de um bem $i$, seu conjunto de características x e um erro ou distorção u.

$$
\mathrm{p}_{\mathrm{i}}=\mathrm{f}\left(\mathrm{x}_{1 \mathrm{i}}, \mathrm{x}_{2 \mathrm{i}, \ldots,} \mathrm{x}_{\mathrm{ki}}, \mathrm{u}\right)
$$

A forma funcional linear $\left(p_{i}=\alpha+\sum_{j} \sum_{k} \beta_{j k} x_{i j k}+u\right)$ é frequentemente usada, onde $\alpha$ e $\beta$ são os coeficientes a serem estimados e $\mathrm{p}_{\mathrm{i}}, \mathrm{x}$ e $\mathrm{u}$, conforme definidos anteriormente. A variável $x_{i j k}$ indica se o k-ésimo nível da j-ésima característica está presente no bem i e o coeficiente $\boldsymbol{\beta}_{j k}$ representa o valor a ser adicionado no preço de um bem que possua o k-ésimo nível da j-ésima característica. Nesse estudo, essa forma funcional foi utilizada, sendo obtida uma regressão linear múltipla com variáveis binárias. 
O critério utilizado para a estimação dos coeficientes foi a minimização do erro quadrático médio (EQM). Após a estimação dos coeficientes, os mesmos foram padronizados de maneira a atribuir peso zero ao menor nível de cada característica (por exemplo, se o menor valor encontrado para um nível de uma determinada característica k é igual a -1, somamos o valor 1 a cada nível da característica $\mathrm{k} \mathrm{e}-1$ à constante $\alpha$, não alterando o valor previsto para o preço $\mathrm{p}$ ). Esse procedimento torna mais simples a visualização dos resultados e facilita o cálculo da importância de cada uma das características. Assim, sendo $\widetilde{\alpha}$ e $\widetilde{\beta}$ os pesos padronizados, temos:

$$
p_{i}=\widetilde{\alpha}+\sum_{j} \sum_{k} \widetilde{\beta}_{j k} x_{i j k}+u, \text { onde } \operatorname{Min}\left(\widetilde{\beta}_{j k}\right)=0, \forall j
$$

Dentre as 150 observações disponíveis para estudo, 38 foram aleatoriamente retiradas para realizar o teste do modelo. Ou seja, as constantes foram estimadas utilizando 112 observações. Posteriormente, foram obtidas previsões para os preços dos 38 automóveis da amostra de teste e compa-

Tabela 2: Descrição das variáveis utilizadas.

\begin{tabular}{|c|c|}
\hline NOME DA VARIÁVEL & DESCRIÇÃO \\
\hline PREÇO & Em reais ( $R \$$ ), atualizado em dezembro/05 \\
\hline MARCA-AUDI & 1 se a marca do carro for Audi, 0 c.c. \\
\hline MARCA-CHEVROLET & 1 se a marca do carro for Chevrolet, 0 c.c. \\
\hline MARCA-CITROËN & 1 se a marca do carro for Citroën, 0 c.c. \\
\hline MARCA-FIAT & 1 se a marca do carro for Fiat, 0 c.c. \\
\hline MARCA-FORD & 1 se a marca do carro for Ford, 0 c.c. \\
\hline MARCA-HONDA & 1 se a marca do carro for Honda, 0 c.c. \\
\hline MARCA-PEUGEOT & 1 se a marca do carro for Peugeot, 0 c.c. \\
\hline MARCA-RENAULT & 1 se a marca do carro for Renault, 0 c.c. \\
\hline MARCA-TOYOTA & 1 se a marca do carro for Toyota, 0 c.c. \\
\hline MARCA-VOLKSWAGEN & 1 se a marca for Volkswagen, 0 c.c. \\
\hline POTÊNCIA $<=55$ & 1 se a potência for $<=55 \mathrm{cv}, 0$ c.c. \\
\hline POTÊNCIA 56-80 & 1 se a potência for $>55 \mathrm{cv} E<=80 \mathrm{cv}, 0$ c.c. \\
\hline POTÊNCIA 81-100 & 1 se a potência for $>80 \mathrm{cv} E<=100 \mathrm{cv}, 0$ c.c. \\
\hline POTÊNCIA 101-115 & 1 se a potência for $>100 \mathrm{cv} E<=115 \mathrm{cv}, 0$ c.c. \\
\hline POTÊNCIA 116-135 & 1 se a potência for $>115 \mathrm{cv} E<=135 \mathrm{cv}, 0$ c.c. \\
\hline POTÊNCIA 136-150 & 1 se a potência for $>135 \mathrm{cv} E<=150 \mathrm{cv}, 0$ c.c. \\
\hline POTÊNCIA $>=151$ & 1 se a potência for $>150 \mathrm{cv}, 0$ c.c. \\
\hline LUXO-1 & 1 se o luxo se enquadrar no nível 1, 0 c.c. \\
\hline LUXO-2 & 1 se o luxo se enquadrar no nível 2, 0 c.c. \\
\hline LUXO-3 & 1 se o luxo se enquadrar no nível 3, 0 c.c. \\
\hline LUXO-4 & 1 se o luxo se enquadrar no nível 4, 0 c.c. \\
\hline LUXO-5 & 1 se o luxo se enquadrar no nível 5, 0 c.c. \\
\hline 3PORTAS & 1 se o carro tem 2 ou 3 portas, 0 c.c. \\
\hline 5PORTAS & 1 se o carro tem 4 ou 5 portas, 0 c.c. \\
\hline COMB-GASOLINA & 1 se o motor do carro é a gasolina, 0 c.c. \\
\hline COMB-FLEX & 1 se o motor do carro é flex, 0 c.c. \\
\hline COMPRIMENTO $<=405$ & 1 se comprimento for $<=405 \mathrm{~cm}, 0$ c.c. \\
\hline COMPRIMENTO >405 E <430 & 1 se comprimento for $>405 \mathrm{~cm} \mathrm{e}<430 \mathrm{~cm}, 0$ c.c. \\
\hline COMPRIMENTO $>=430$ & 1 se comprimento for $>=430 \mathrm{~cm}, 0$ c.c. \\
\hline
\end{tabular}


radas com os valores reais. Todos os cálculos foram feitos utilizando a função "Im" do software R (R development core team, 2005).

\subsection{Resultados e discussão}

A Tabela 3 apresenta o resumo dos resultados da regressão hedônica linear.

O modelo obtido apresentou coeficiente de determinação R2 igual a 94,5\%. A estatística de teste $\mathrm{F}$ obtida foi igual a 65,72 , indicando, com forte evidência ( $p$-value $<0,001$ ), que podemos rejeitar a hipótese de que os coeficientes do modelo são conjuntamente nulos.

Na Figura 2 temos a comparação entre as curvas dos preços reais dos veículos utilizados para a construção do modelo e a curva aproximada pelo modelo.

Na Figura 3 segue a comparação entre as curvas dos preços reais dos veículos separados para teste e a curva aproximada pelo modelo. O erro padrão residual para os dados utilizados no teste do modelo foi aproximadamente $\mathrm{R} \$ 3.957$.

Pode-se observar que o modelo foi capaz de representar com boa precisão os preços dos veículos nacionais, uma vez que a regressão apresentou um alto valor de R2 ajustado $(93,1 \%)$ e o erro padrão residual obtido para os dados de teste ( $R \$ 3.957)$ foi bem próximo do erro pa- drão residual estimado no desenvolvimento do modelo ( R\$ 4.445).

Os valores calculados para cada nível das seis características consideradas são mostrados nas Figuras 4 a 9.

Analisando os pesos obtidos por marca, percebe-se que a marca AUDI é aquela que acrescenta maior preço a um veículo ( $R \$ 18.433$ ), o que é razoável por se tratar de uma marca que explicitamente tenta conquistar seus clientes oferecendo status e diferenciação. A marca HONDA é aquela que menos impacta o preço de um veículo, o que também é razoável por se tratar de uma marca que se destaca por menores custos de produção.

Os pesos obtidos por potência foram conforme o esperado, sendo que o peso mínimo obtido foi para potência menor do que $55 \mathrm{cv}$ e o máximo de $\mathrm{R} \$ 29.593$ para potência maior do que $150 \mathrm{cv}$. O grande valor encontrado para esse nível de potência sinaliza a grande importância da potência na precificação dos veículos nacionais.

Os pesos obtidos por luxo também foram conforme o esperado, sendo que o peso mínimo obtido para o nível 1 de luxo (básico) e o máximo de $\mathrm{R} \$ 28.024$ para o nível 5 de luxo (completo com câmbio automático). O grande valor encontrado para esse nível de luxo sinaliza a grande importância do luxo na precificação de veículos.

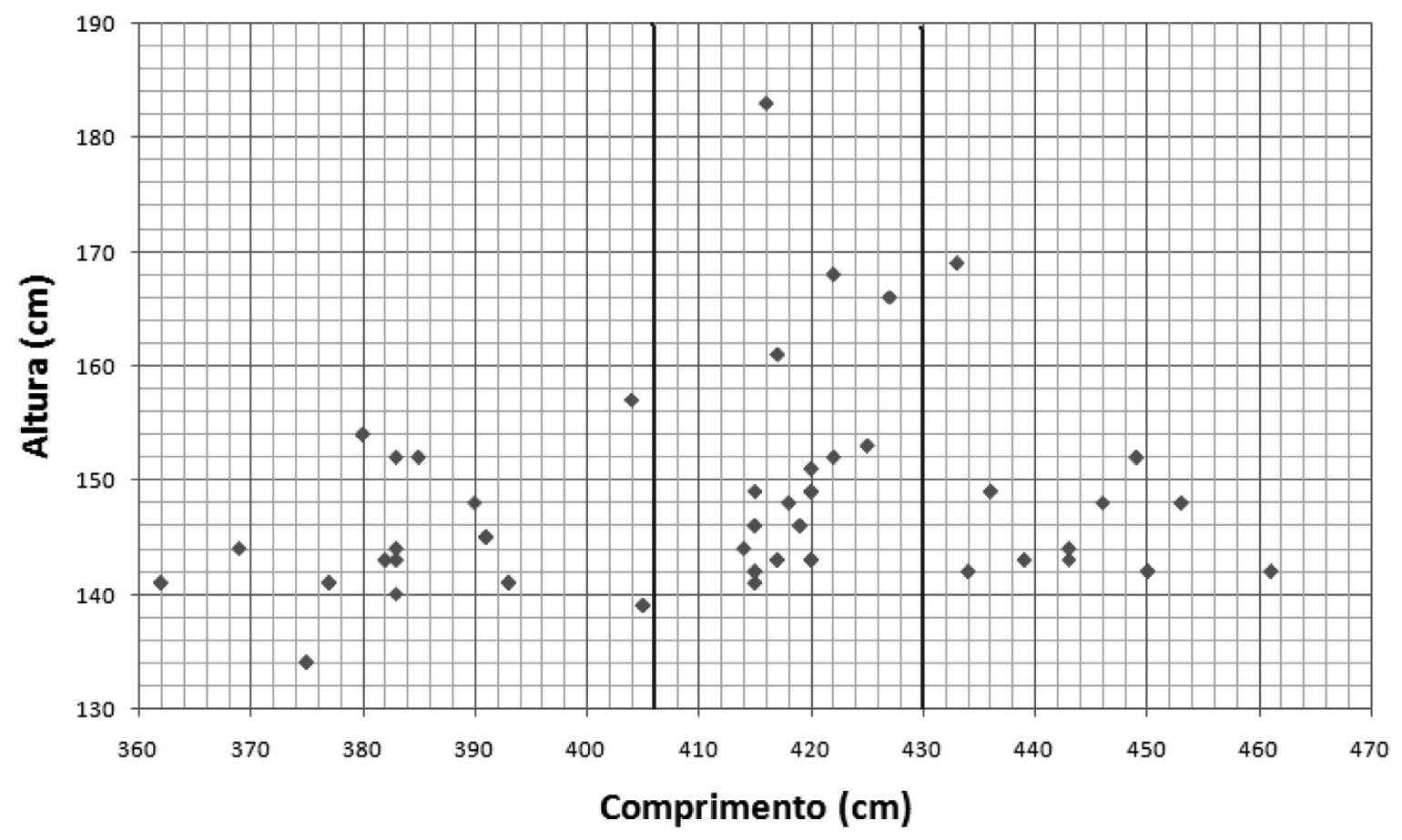

Figura 1: Gráfico utilizado para escolha de faixas de comprimento. 
O peso obtido para tipo de combustível flex (R\$ 80) é muito pequeno quando comparado a qualquer outra característica, o que indica que o tipo de combustível não é um fator determinante na precificação de automóveis.

Os pesos obtidos por comprimento também foram conforme o esperado, sendo que o peso mínimo obtido foi para veículos com comprimento menor do que $405 \mathrm{~cm}$ e o máximo de $\mathrm{R} \$$ 6.866 para veículos com comprimento maior do que $430 \mathrm{~cm}$.

Tabela 3: Valores calculados para os coeficientes normalizados.

\begin{tabular}{|c|c|c|c|}
\hline \multicolumn{2}{|c|}{ COEFICIENTES } & \multirow{2}{*}{$\begin{array}{c}\begin{array}{c}\text { VALOR CALCULADO } \\
(\mathrm{R} \$)\end{array} \\
14410,21\end{array}$} & \multirow{2}{*}{$\begin{array}{c}\begin{array}{c}\text { ESTATÍSTICA } \\
\mathrm{t}\end{array} \\
3,494\end{array}$} \\
\hline & INTERCEPTO & & \\
\hline \multirow{10}{*}{ MARCA } & AUDI & $18.433,30$ & 5,747 \\
\hline & CHEVROLET & $3.079,22$ & 1,304 \\
\hline & CITROËN & $2.186,26$ & 0,716 \\
\hline & FIAT & $3.700,88$ & 1,460 \\
\hline & FORD & $2.242,86$ & 0,906 \\
\hline & HONDA & 0 & - \\
\hline & PEUGEOT & $3.253,88$ & 1,096 \\
\hline & RENAULT & 310,57 & 0,119 \\
\hline & TOYOTA & $2.137,10$ & 0,598 \\
\hline & VOLKSWAGEN & $2.704,59$ & 1,087 \\
\hline \multirow[t]{7}{*}{ POTÊNCIA (cv) } & $<=55$ & 0 & - \\
\hline & $56-80$ & $5.216,85$ & 1,477 \\
\hline & $81-100$ & $6.148,46$ & 1,572 \\
\hline & $101-115$ & $10.296,01$ & 2,716 \\
\hline & 116-135 & $16.223,54$ & 4,047 \\
\hline & $136-150$ & $21.186,61$ & 5,065 \\
\hline & $>=151$ & $29.593,05$ & 5,714 \\
\hline \multirow[t]{5}{*}{ LUXO } & 1 - Básico & 0 & - \\
\hline & 2 & $4.472,40$ & 3,133 \\
\hline & 3- $\mathrm{Ar}+\mathrm{DH}$ & $12.898,50$ & 8,255 \\
\hline & 4 & $21.150,03$ & 9,430 \\
\hline & 5 - Completo & $28.024,37$ & 10,960 \\
\hline \multirow[t]{2}{*}{$\mathrm{N}^{0}$ PORTAS } & 3 & 0 & - \\
\hline & 5 & $1.589,11$ & 1,710 \\
\hline \multirow[t]{2}{*}{ COMBUSTÍVEL } & GASOLINA & 0 & - \\
\hline & FLEX & 80,19 & 0,069 \\
\hline \multirow{3}{*}{$\begin{array}{l}\text { COMPRIMENTO } \\
\text { (cm) }\end{array}$} & $<=405$ & 0 & - \\
\hline & $>405 \mathrm{E}<430$ & $3.893,93$ & 3,124 \\
\hline & $>=430$ & $6.866,34$ & 3,902 \\
\hline
\end{tabular}

Na Figura 10, segue a comparação entre os valores calculados para todos os níveis possíveis de todas as características consideradas.

Depois de estimados os pesos de cada nível de cada uma das características, foi determinada a importância relativa de cada característica. A importância relativa de uma característica foi considerada como a razão entre o maior peso estimado para um nível daquela característica e a soma dos maiores pesos de cada uma das características. Os resultados obtidos para as importâncias relativas das características são exibidos na Tabela 4 e na Figura 11.

Analisando os valores obtidos para as importâncias relativas na precificação, percebe-se claramente que três fatores são os mais importantes para determinação de preço: potência $(34,5 \%)$, luxo $(32,7 \%)$ e marca $(21,5 \%)$. Esses três fatores juntos corresponderam a $88,7 \%$ de importância no preço. O baixo valor obtido para a importância do tipo de combustível $(0,1 \%)$ confirma que realmente essa não é uma característica de importância considerável na precificação de automóveis.

\section{SIMULAÇÃO DE APLICAÇÃO DO MODELO}

Para simular uma aplicação do modelo matemático desenvolvido, estimou-se o preço de um veículo nacional lançado após a coleta de dados e comparou-se a estimativa com o preço real de lançamento (preço sem frete informado no web-site oficial da montadora em outubro de 2006).

$\mathrm{O}$ veículo analisado foi um Chevrolet Vectra (Modelo Elite). A Tabela 5 apresenta as características do veículo extraídas do web-site da montadora.

Realizando a simulação, foram obtidos os resultados mostrados na Tabela 6 .

O valor estimado foi bem próximo do valor real, apresentando um erro de estimativa menor do que 0,5\% em módulo.

\section{CONCLUSÕES}

Através do modelo obtido pode-se perceber a grande utilidade de regressões hedônicas para análise de preços em mercados com produtos diferenciados. Utilizando-se um simples modelo de regressão hedônica linear, foi possível determinar os fatores que mais influenciam os preços dos veículos nacionais e, ainda, construir um modelo de previsão de preços de novos produtos.

Pode-se concluir que o fator de maior impacto no preço de um veículo nacional é a potência do motor (34,5\%), seguido pelo grau de luxo $(32,7 \%)$ e marca $(21,5 \%)$. Outra conclusão importante é que o tipo de combustível não é um fator determinante na precificação dos veículos nacionais. 


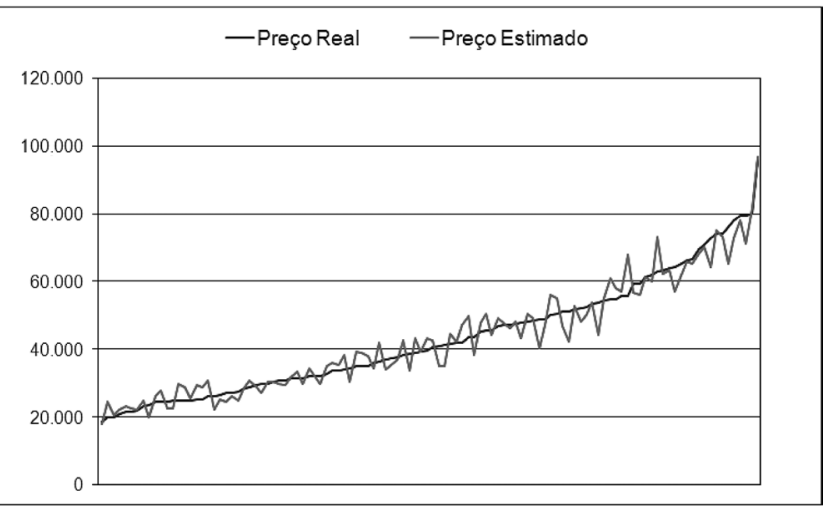

Figura 2: Comparação entre valores estimados pelo modelo e valores reais (amostra utilizada na estimação dos parâmetros).

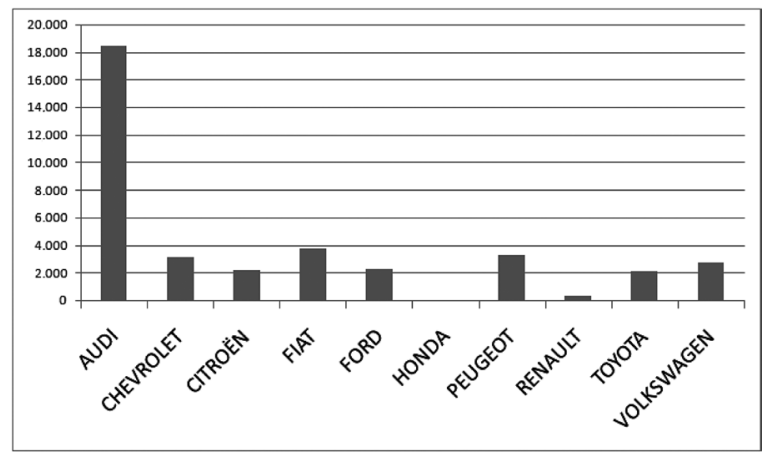

Figura 4: Valores calculados (R\$̣) para o peso de cada marca.

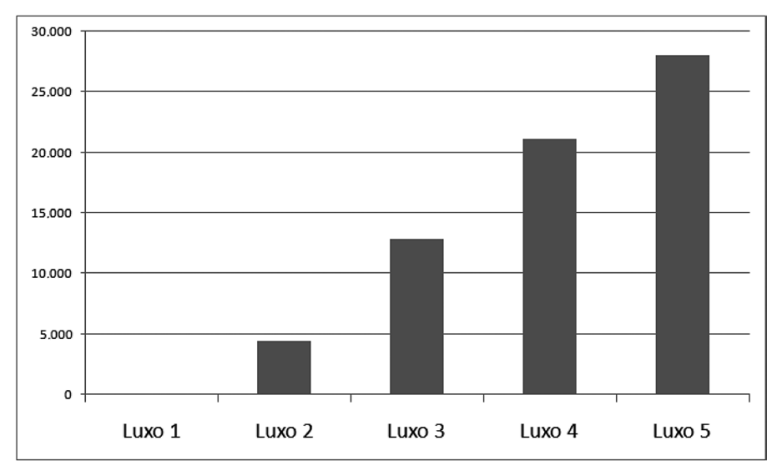

Figura 6: Valores calculados (R\$) para o peso de cada faixa de luxo.

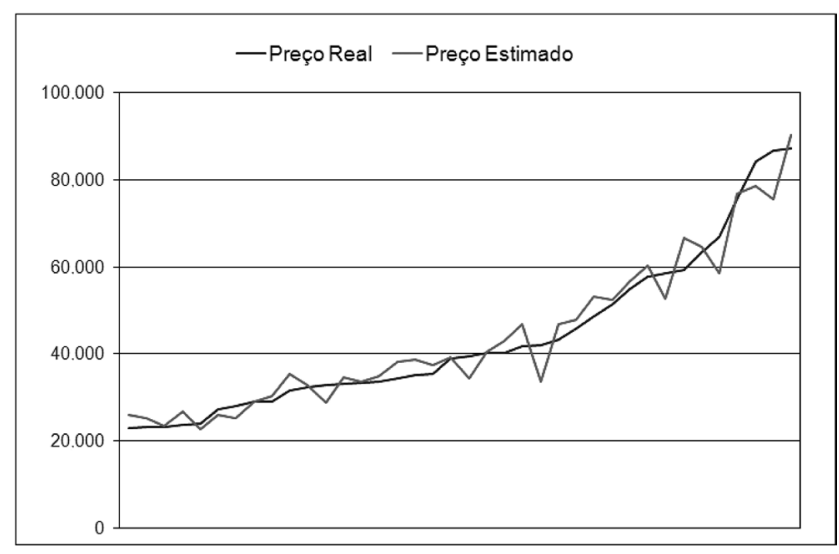

Figura 3: Comparação entre valores estimados pelo modelo e valores reais (amostra de teste).

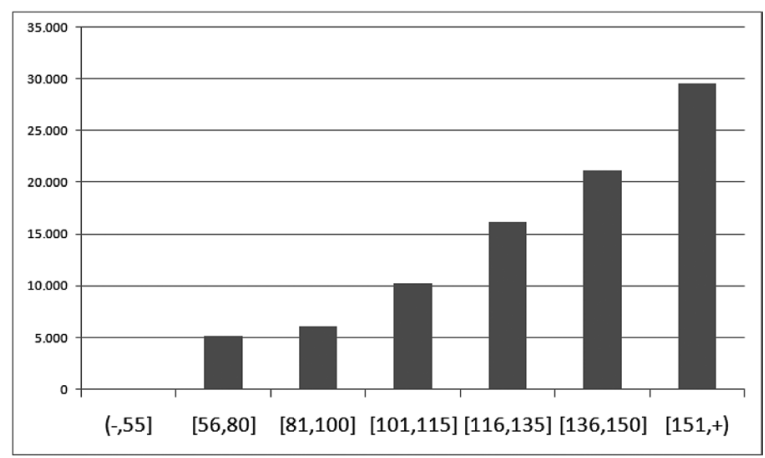

Figura 5: Valores calculados (R\$̦) para o peso de cada faixa de potência (cv).

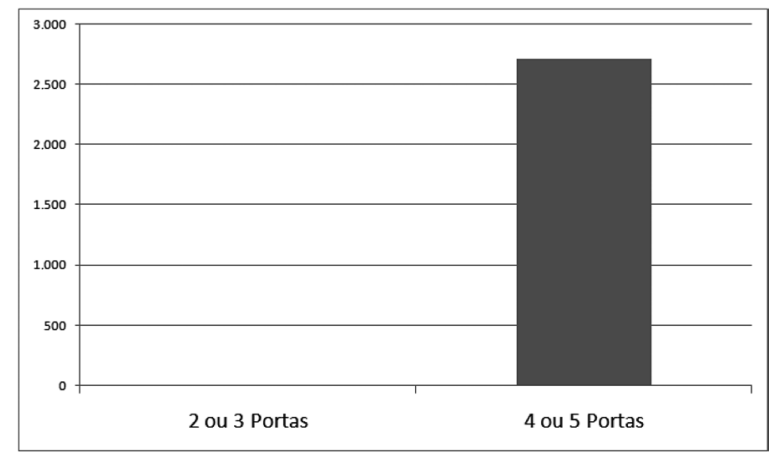

Figura 7: Valores calculados (R\$̦) para o peso do número de portas. 


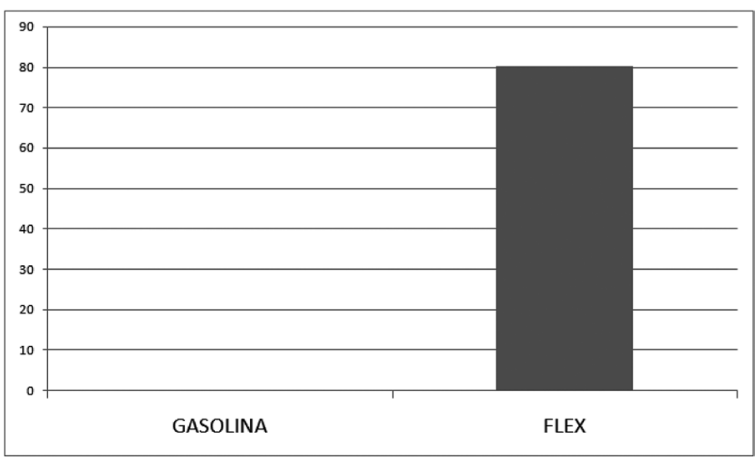

Figura 8: Valores calculados (R\$̦) para o peso de cada tipo de combustível.

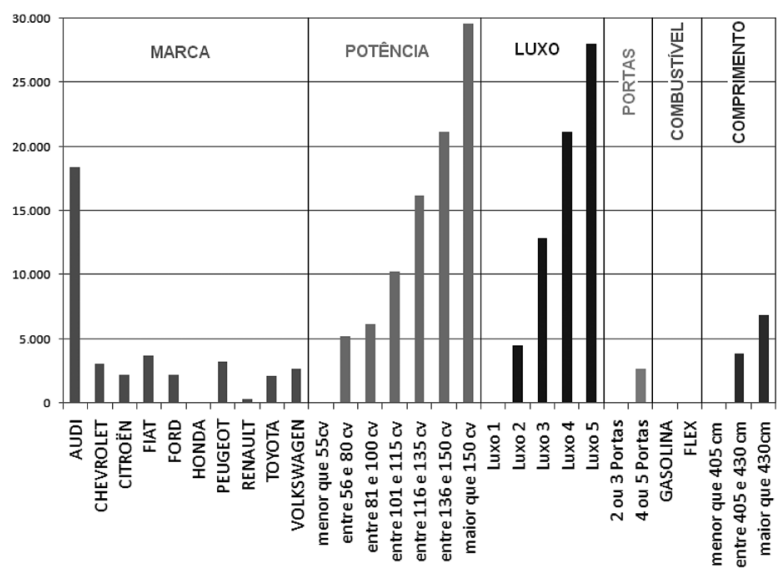

Figura 10: Comparação entre os valores calculados $(R \$)$ para os níveis de diferentes características.

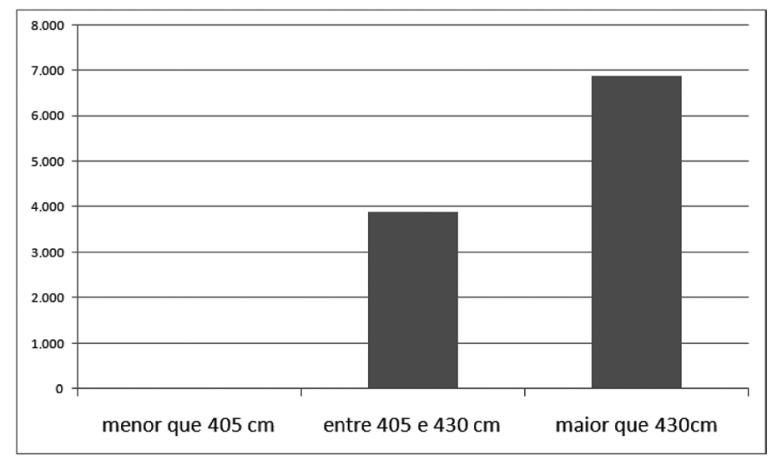

Figura 9: Valores calculados (R\$̣) para o peso de cada faixa de comprimento.

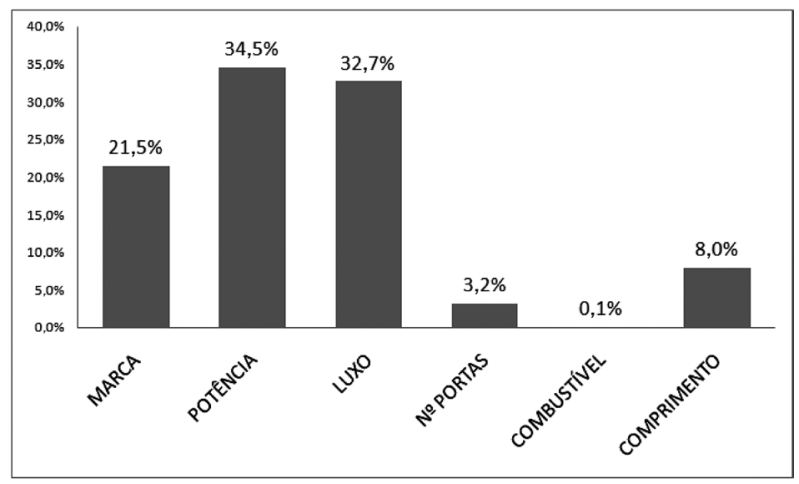

Figura 11: Importância de cada característica na precificação dos veículos nacionais novos.

Tabela 4: Cálculo das importâncias relativas de cada característica na precificação.

\begin{tabular}{ccc}
\hline CARACTERÍSTICA & MAIOR PESO ESTIMADO (R\$) & IMPORTÂNCIA RELATIVA (\%) \\
\hline MARCA & 18.433 & 21,5 \\
POTÊNCIA & 29.593 & 34,5 \\
LUXO & 28.024 & 32,7 \\
N ${ }^{0}$ PORTAS & 2.718 & 3,2 \\
COMBUSTÍVEL & 80 & 0,1 \\
COMPRIMENTO & 6.866 & 8,0 \\
\hline
\end{tabular}

Tabela 5: Dados extraídos para realização de simulação de aplicação.

\begin{tabular}{cccccc}
\hline MODELO & POTÊNCIA (cv) & COMPRIMENTO (cm) & LUXO & PREÇO (RȘ) & No DE PORTAS $^{\circ}$ \\
\hline VECTRA ELITE & 151 & 462 & 5 & 84.990 & 4 \\
\hline
\end{tabular}


Tabela 6: Resultados da simulação e comparação com os preços reais.

\begin{tabular}{cccc}
\hline MODELO & PREÇO (R\$) & PREÇO ESTIMADO (R\$) & ERRO (\%) \\
\hline VECTRA ELITE & 84.990 & 84.691 & $-0,4$ \\
\hline
\end{tabular}

\section{Artigo recebido em 29/01/2008 Aprovado para publicação em 26/03/2009}

\section{REFERÊNCIAS}

AGARWAL, M. K.; RATCHFORD, B. T. Estimating demand functions for product characteristics: the case of automobiles. Journal of Consumer Research, v. 8, p. 249-262, 1980.

ARGUA, N. M.; HSIAO, C.; TAYLOR, G. A. Estimating consumer preferences using market data: An application to US automobile demand. Journal of Applied Econometrics, v. 9 , p. 1-18, 1994.

BOSKIN, M. J. et al. Toward a more accurate measure of the cost of living. Final Report to the Senate Finance Committee from the Advisory Commission to Study the Consumer Price Index. 1996.

BRACHINGER, H. W. Statistical Theory of Hedonic Price Indices. Working Paper (to appear in: BRACHINGER, H. W.; DIEWERT, E. HEDONIC. Methods in Price Statistics: Theory and Practice. Springer, Heidelberg), 2002.

COLWELL, P. F.; DILMORE, G. Who was first? An examination of an early hedonic study, Land Economics, v. 75, n. 4, p. 620-626, 1999.
GOODMAN, A. C. Andrew court and the invention of hedonic price analysis. Journal of Urban Economics, v. 44, p. 291-298, 1998.

GRILICHES, Z. Hedonic price indexes for automobiles: An econometric analysis of quality change. In: GRILICHES, Z. Price indexes and quality change: studies in new methods of measurement. Cambridge: Harvard University Press, p. 55-87, 1961.

LANCASTER, K. A new approach to consumer's theory. Journal of Political Economy, v. 74, p. 132-157, 1966

LINZ, S. Hedonic price measurements for IT products. Statistisches Bundesamt, 2004.

NERLOVE, M. Hedonic price functions and the measurement of preferences: the case of Swedish wine consumers. European Economic Review, v. 39, p. 1697-1716, 1995.
NETO, E. Estimação do preço hedônico: uma aplicação para o mercado imobiliário da cidade do Rio de Janeiro. 2002. Dissertação (Mestrado em Economia), Fundação Getúlio Vargas, Rio de Janeiro.

PAKES, A. A reconsideration of hedonic price indexes with an application to PC's. American Economic Review, v. 93, p. 1578-1614, 2003.

R DEVELOPMENT CORE TEAM. R: A language and environment for statistical computing. $\mathrm{R}$ Foundation for Statistical Computing, Vienna, Austria. ISBN 3-900051-07-0, URL: http:// www.R-project.org, 2005.

REIS, H. J.; SILVA, J. M. C. Hedonic prices indexes for new passenger cars in Portugal (1997-2001). Economic Modelling, v. 23, p. 890-908, 2006.

ROSEN, S. Hedonic price and implicit markets: product differentiation in pure competition. Journal of Political Economy, v. 82, p. 34-55, 1974.

\section{AGRADECIMENTOS}

Os autores agradecem aos revisores anônimos pelas sugestões e à FAPESP pelo apoio financeiro.

\section{SOBRE OS AUTORES}

\section{Camilo Brandão de Resende}

Instituto Tecnológico de Aeronáutica (ITA)

End.: Pça. Mal. Eduardo Gomes, 50 sala 2311 - São José dos Campos - SP - 12228-900

E-mail: camilo@ita.br

\section{Rodrigo Arnaldo Scarpel}

Instituto Tecnológico de Aeronáutica (ITA)

End.: Pça. Mal. Eduardo Gomes, 50 sala 2311 - São José dos Campos - SP - 12228-900

End.: rodrigo@ita.br 


\section{APÊNDICE A: DADOS UTILIZADOS NO TRABALHO}

TABELA 7: Dados utilizados na estimação dos coeficientes do modelo.

\begin{tabular}{|c|c|c|c|c|c|c|c|c|c|c|}
\hline MOD & MAR & COMB & POT & PM & COMP & ALT & LUX & CAM & PREÇO & NP \\
\hline UNO & FIAT & GAS & 55 & 222 & 369 & 144 & 1 & MEC & 18.780 & 3 \\
\hline$K A$ & FORD & GAS & 65 & 184 & 362 & 141 & 1 & MEC & 20.060 & 5 \\
\hline UNO & FIAT & GAS & 55 & 222 & 369 & 144 & 1 & MEC & 20.160 & 5 \\
\hline GOL & VW & GAS & 57 & 246 & 393 & 141 & 1 & MEC & 20.971 & 3 \\
\hline PALIO & FIAT & GAS & 65 & 246 & 383 & 144 & 1 & MEC & 21.580 & 3 \\
\hline CELTA & CHEV & GAS & 70 & 243 & 375 & 134 & 1 & MEC & 21.670 & 3 \\
\hline GOL & VW & GAS & 65 & 246 & 393 & 141 & 1 & MEC & 22.052 & 3 \\
\hline GOL & VW & GAS & 65 & 246 & 393 & 141 & 1 & MEC & 23.224 & 5 \\
\hline CLIO & REN & GAS & 58 & 255 & 377 & 141 & 1 & MEC & 23.590 & 3 \\
\hline CELTA & CHEV & GAS & 85 & 243 & 375 & 134 & 1 & MEC & 24.540 & 5 \\
\hline CELTA & CHEV & GAS & 85 & 243 & 375 & 134 & 2 & MEC & 24.820 & 3 \\
\hline CLIO & REN & GAS & 58 & 255 & 377 & 141 & 1 & MEC & 24.840 & 5 \\
\hline CLASSIC & CHEV & GAS & 70 & 345 & 405 & 139 & 1 & MEC & 24955 & 3 \\
\hline SIENA & FIAT & GAS & 65 & 441 & 414 & 144 & 1 & MEC & 24.990 & 5 \\
\hline FIESTA STR. & FORD & GAS & 65 & 208 & 383 & 140 & 2 & MEC & 25.020 & 5 \\
\hline 206 & PEUG & GAS & 70 & 222 & 383 & 143 & 1 & MEC & 25.090 & 5 \\
\hline GOL & VW & GAS & 76 & 246 & 393 & 141 & 2 & MEC & 25.304 & 5 \\
\hline $\mathrm{KA}$ & FORD & GAS & 65 & 184 & 362 & 141 & 2 & MEC & 25.330 & 5 \\
\hline CELTA & CHEV & GAS & 85 & 243 & 375 & 134 & 2 & MEC & 26.230 & 5 \\
\hline FOX & VW & FX & 72 & 257 & 380 & 154 & 1 & MEC & 26.456 & 3 \\
\hline CORSA & CHEV & GAS & 71 & 236 & 382 & 143 & 1 & MEC & 26.760 & 5 \\
\hline FIESTA & FORD & GAS & 66 & 255 & 391 & 145 & 1 & MEC & 27.220 & 5 \\
\hline CLASSIC & CHEV & GAS & 92 & 345 & 405 & 139 & 1 & MEC & 27.407 & 5 \\
\hline FOX & VW & FX & 72 & 257 & 380 & 154 & 1 & MEC & 27.781 & 5 \\
\hline FIESTA SEDAN & FORD & GAS & 66 & 492 & 420 & 149 & 1 & MEC & 28.580 & 5 \\
\hline CLIO SEDAN & REN & GAS & 70 & 484 & 415 & 141 & 2 & MEC & 28.810 & 5 \\
\hline FIESTA SEDAN & FORD & GAS & 95 & 492 & 420 & 149 & 1 & MEC & 29.580 & 5 \\
\hline 206 & PEUG & GAS & 75 & 222 & 383 & 143 & 2 & MEC & 29.840 & 3 \\
\hline CORSA & CHEV & FX & 107 & 260 & 382 & 143 & 1 & MEC & 29.920 & 5 \\
\hline GOL & VW & FX & 98 & 246 & 393 & 141 & 2 & MEC & 30.510 & 5 \\
\hline 206 & PEUG & GAS & 75 & 222 & 383 & 143 & 2 & MEC & 30.940 & 5 \\
\hline FIESTA & FORD & FX & 108 & 255 & 391 & 145 & 1 & MEC & 31.030 & 5 \\
\hline FOX & VW & FX & 102 & 257 & 380 & 154 & 2 & MEC & 31.560 & 3 \\
\hline FIESTA SEDAN & FORD & FX & 108 & 492 & 420 & 149 & 1 & MEC & 31.640 & 5 \\
\hline SIENA & FIAT & FX & 70 & 441 & 414 & 144 & 1 & MEC & 31.750 & 5 \\
\hline CORSA SEDAN & CHEV & FX & 107 & 435 & 417 & 143 & 1 & MEC & 32.115 & 5 \\
\hline CLIO & REN & FX & 113 & 255 & 377 & 141 & 2 & MEC & 32.150 & 5 \\
\hline FIESTA & FORD & GAS & 95 & 255 & 391 & 145 & 2 & MEC & 32.390 & $\begin{array}{c}5 \\
\text { (continua) }\end{array}$ \\
\hline
\end{tabular}




\begin{tabular}{|c|c|c|c|c|c|c|c|c|c|}
\hline CORSA & CHEV & FX & 107 & 260 & 382 & 143 & 2 & MEC & 32.825 \\
\hline CLIO SEDAN & REN & FX & 113 & 484 & 415 & 141 & 2 & MEC & 33.820 \\
\hline PALIO & FIAT & GAS & 103 & 246 & 383 & 144 & 2 & MEC & 33.920 \\
\hline $\mathrm{KA}$ & FORD & GAS & 95 & 184 & 362 & 141 & 3 & MEC & 34.130 \\
\hline PALIO & FIAT & FX & 70 & 246 & 383 & 144 & 2 & MEC & 34.570 \\
\hline SIENA & FIAT & FX & 108 & 441 & 414 & 144 & 2 & MEC & 35.050 \\
\hline CLASSIC & CHEV & GAS & 92 & 345 & 405 & 139 & 3 & MEC & 35.241 \\
\hline FIESTA SEDAN & FORD & FX & 108 & 492 & 420 & 149 & 2 & MEC & 35.320 \\
\hline PARATI & VW & FX & 98 & 443 & 419 & 146 & 2 & MEC & 36.274 \\
\hline CORSA SEDAN & CHEV & GAS & 71 & 435 & 417 & 143 & 3 & MEC & 36.580 \\
\hline $\mathrm{C} 3$ & CITR & GAS & 110 & 293 & 385 & 152 & 2 & MEC & 37.110 \\
\hline CLIO & REN & GAS & 70 & 255 & 377 & 141 & 3 & MEC & 37.530 \\
\hline SANTANA & VW & GAS & 99 & 413 & 461 & 142 & 2 & MEC & 37.844 \\
\hline FIESTA & FORD & FX & 108 & 255 & 391 & 145 & 3 & MEC & 38.590 \\
\hline ECOSPORT & FORD & GAS & 95 & 288 & 422 & 168 & 2 & MEC & 38.760 \\
\hline 206 & PEUG & GAS & 110 & 222 & 383 & 143 & 3 & MEC & 39.100 \\
\hline PALIO WK & FIAT & FX & 108 & 402 & 422 & 152 & 2 & MEC & 39.570 \\
\hline CORSA & CHEV & FX & 107 & 260 & 382 & 143 & 3 & MEC & 39.960 \\
\hline PARATI & VW & FX & 98 & 443 & 419 & 146 & 3 & MEC & 40.943 \\
\hline MERIVA & CHEV & FX & 107 & 339 & 404 & 157 & 2 & MEC & 41.275 \\
\hline FIT & HOND & GAS & 80 & 355 & 383 & 152 & 3 & MEC & 41.435 \\
\hline CLIO SEDAN & REN & FX & 113 & 484 & 415 & 141 & 3 & MEC & 41.700 \\
\hline $\mathrm{C} 3$ & CITR & GAS & 110 & 293 & 385 & 152 & 3 & MEC & 41.960 \\
\hline CORSA SEDAN & CHEV & FX & 107 & 435 & 417 & 143 & 3 & MEC & 42.160 \\
\hline SANTANA & VW & GAS & 114 & 413 & 461 & 142 & 3 & MEC & 43.726 \\
\hline GOLF & VW & GAS & 101 & 336 & 415 & 146 & 2 & MEC & 43.868 \\
\hline STILO & FIAT & GAS & 103 & 380 & 425 & 153 & 3 & MEC & 45.390 \\
\hline ASTRA & CHEV & FX & 125 & 332 & 420 & 143 & 3 & MEC & 45.603 \\
\hline GOLF & VW & GAS & 116 & 336 & 415 & 146 & 2 & MEC & 45.723 \\
\hline FOCUS SEDAN & FORD & GAS & 103 & 460 & 436 & 149 & 3 & MEC & 46.870 \\
\hline DOBLÒ & FIAT & GAS & 103 & 584 & 416 & 183 & 3 & MEC & 47.500 \\
\hline FOCUS & FORD & GAS & 103 & 285 & 415 & 149 & 3 & MEC & 47.515 \\
\hline MAREA & FIAT & GAS & 132 & 409 & 439 & 143 & 2 & MEC & 47.790 \\
\hline MERIVA & CHEV & FX & 107 & 339 & 404 & 157 & 3 & MEC & 47.895 \\
\hline $\mathrm{C} 3$ & CITR & GAS & 110 & 293 & 385 & 152 & 4 & MEC & 48.400 \\
\hline COROLLA & TOYO & GAS & 110 & 461 & 453 & 148 & 3 & MEC & 48.612 \\
\hline FIT & HOND & GAS & 105 & 355 & 383 & 152 & 3 & MEC & 49.065 \\
\hline 307 & PEUG & GAS & 110 & 420 & 420 & 151 & 3 & MEC & 49.100 \\
\hline ASTRA SEDAN & CHEV & FX & 125 & 460 & 434 & 142 & 3 & MEC & 50.190 \\
\hline GOLF & VW & GAS & 101 & 336 & 415 & 146 & 4 & MEC & 50.628 \\
\hline CIVIC & HOND & GAS & 115 & 395 & 443 & 144 & 3 & MEC & 51.165 \\
\hline
\end{tabular}




\begin{tabular}{|c|c|c|c|c|c|c|c|c|c|}
\hline ECOSPORT & FORD & GAS & 98 & 288 & 422 & 168 & 3 & MEC & 51.390 \\
\hline POLO SEDAN & VW & GAS & 116 & 409 & 418 & 148 & 3 & MEC & 52.086 \\
\hline MAREA WK & FIAT & GAS & 132 & 500 & 449 & 152 & 2 & MEC & 52.340 \\
\hline VECTRA & CHEV & GAS & 110 & 402 & 450 & 142 & 3 & MEC & 52.694 \\
\hline STILO & FIAT & GAS & 122 & 380 & 425 & 153 & 3 & MEC & 53.450 \\
\hline SCÉNIC & REN & GAS & 110 & 396 & 417 & 161 & 3 & MEC & 53.990 \\
\hline FOCUS SEDAN & FORD & GAS & 126 & 460 & 436 & 149 & 3 & MEC & 54.620 \\
\hline CIVIC & HOND & GAS & 130 & 395 & 443 & 144 & 4 & MEC & 54.780 \\
\hline ASTRA & CHEV & GAS & 136 & 332 & 420 & 143 & 3 & MEC & 55.053 \\
\hline PICASSO & CITR & GAS & 138 & 450 & 427 & 166 & 3 & MEC & 55.830 \\
\hline CIVIC & HOND & GAS & 130 & 395 & 443 & 144 & 5 & AUTO & 55.920 \\
\hline MAREA WK & FIAT & GAS & 132 & 500 & 449 & 152 & 3 & MEC & 59.480 \\
\hline ZAFIRA & CHEV & FX & 125 & 535 & 433 & 169 & 3 & MEC & 59.641 \\
\hline ASTRA & CHEV & $\mathrm{FX}$ & 125 & 332 & 420 & 143 & 4 & MEC & 61.495 \\
\hline FIELDER & TOYO & GAS & 136 & 395 & 446 & 148 & 3 & MEC & 61.984 \\
\hline MÉGANE SEDAN & REN & GAS & 138 & 510 & 443 & 143 & 5 & AUTO & 62.990 \\
\hline A3 & AUDI & GAS & 101 & 303 & 415 & 142 & 3 & MEC & 63.341 \\
\hline SCÉNIC & REN & GAS & 140 & 396 & 417 & 161 & 4 & MEC & 64.090 \\
\hline ECOSPORT & FORD & GAS & 143 & 288 & 422 & 168 & 3 & MEC & 64.500 \\
\hline STILO & FIAT & GAS & 122 & 380 & 425 & 153 & 4 & MEC & 65.520 \\
\hline GOLF & VW & GAS & 150 & 336 & 415 & 146 & 4 & MEC & 66.409 \\
\hline PICASSO & CITR & GAS & 138 & 450 & 427 & 166 & 4 & AUTO & 66.890 \\
\hline $\mathrm{A} 3$ & AUDI & GAS & 125 & 303 & 415 & 142 & 3 & MEC & 69.698 \\
\hline SCÉNIC & REN & GAS & 140 & 396 & 417 & 161 & 5 & AUTO & 70.990 \\
\hline ZAFIRA & CHEV & $\mathrm{FX}$ & 125 & 535 & 433 & 169 & 4 & MEC & 72.968 \\
\hline COROLLA & TOYO & GAS & 136 & 461 & 453 & 148 & 5 & AUTO & 74.289 \\
\hline A3 & AUDI & GAS & 150 & 303 & 415 & 142 & 3 & MEC & 74.355 \\
\hline VECTRA & CHEV & GAS & 110 & 402 & 450 & 142 & 5 & MEC & 76.266 \\
\hline GOLF & VW & GAS & 150 & 336 & 415 & 146 & 5 & AUTO & 78.348 \\
\hline MAREA & FIAT & GAS & 160 & 409 & 439 & 143 & 4 & MEC & 79.540 \\
\hline ZAFIRA & CHEV & FX & 125 & 535 & 433 & 169 & 5 & MEC & 79.676 \\
\hline A3 & AUDI & GAS & 150 & 303 & 415 & 142 & 4 & AUTO & 80.267 \\
\hline A3 & AUDI & GAS & 180 & 303 & 415 & 142 & 5 & AUTO & 95.971 \\
\hline
\end{tabular}

TABELA 8: Dados utilizados no teste do modelo.

\begin{tabular}{cccccccccccc}
\hline MOD & MAR & COMB & POT & PM & COMP & ALT & LUX & CAM & PREÇO & NP \\
\hline PALIO & FIAT & GAS & 65 & 246 & 383 & 144 & 1 & MEC & 22.930 & 5 \\
CELTA & CHEV & GAS & 70 & 243 & 375 & 134 & 1 & MEC & 23.080 & 5 \\
CELTA & CHEV & GAS & 85 & 243 & 375 & 134 & 1 & MEC & 23.150 & 3 \\
GOL & VW & GAS & 76 & 246 & 393 & 141 & 2 & MEC & 23.785 & 3 \\
& & & & & & & & & & &
\end{tabular}




\begin{tabular}{|c|c|c|c|c|c|c|c|c|c|c|}
\hline 206 & PEUG & GAS & 70 & 222 & 383 & 143 & 1 & MEC & 23.990 & 3 \\
\hline GOL & VW & FX & 98 & 246 & 393 & 141 & 1 & MEC & 27.272 & 5 \\
\hline FIESTA & FORD & GAS & 95 & 255 & 391 & 145 & 1 & MEC & 27.910 & 5 \\
\hline CORSA SEDAN & CHEV & GAS & 71 & 435 & 417 & 143 & 1 & MEC & 28.905 & 5 \\
\hline PALIO & FIAT & $\mathrm{FX}$ & 70 & 246 & 383 & 144 & 2 & MEC & 28.920 & 5 \\
\hline CLASSIC & CHEV & GAS & 70 & 345 & 405 & 139 & 3 & MEC & 31.600 & 3 \\
\hline FIESTA SEDAN & FORD & GAS & 66 & 492 & 420 & 149 & 2 & MEC & 32.420 & 5 \\
\hline $\mathrm{C} 3$ & CITR & GAS & 75 & 293 & 385 & 152 & 2 & MEC & 32.900 & 5 \\
\hline FOX & VW & FX & 102 & 257 & 380 & 154 & 2 & MEC & 32.957 & 5 \\
\hline FIESTA SEDAN & FORD & GAS & 95 & 492 & 420 & 149 & 2 & MEC & 33.370 & 5 \\
\hline 206 & PEUG & GAS & 110 & 222 & 383 & 143 & 2 & MEC & 33.550 & 5 \\
\hline CORSA & CHEV & GAS & 71 & 260 & 382 & 143 & 3 & MEC & 34.430 & 5 \\
\hline CORSA SEDAN & CHEV & FX & 107 & 435 & 417 & 143 & 2 & MEC & 35.020 & 5 \\
\hline FIESTA & FORD & GAS & 66 & 255 & 391 & 145 & 3 & MEC & 35.450 & 5 \\
\hline CLIO SEDAN & REN & GAS & 70 & 484 & 415 & 141 & 3 & MEC & 39.000 & 5 \\
\hline DOBLÒ & FIAT & GAS & 80 & 584 & 416 & 183 & 2 & MEC & 39.380 & 5 \\
\hline CLIO & REN & FX & 113 & 255 & 377 & 141 & 3 & MEC & 40.230 & 5 \\
\hline POLO & VW & $\mathrm{FX}$ & 102 & 270 & 390 & 148 & 3 & MEC & 40.280 & 5 \\
\hline PARATI & VW & GAS & 112 & 443 & 419 & 146 & 3 & MEC & 41.647 & 5 \\
\hline ECOSPORT & FORD & GAS & 98 & 288 & 422 & 168 & 2 & MEC & 41.950 & 5 \\
\hline POLO SEDAN & VW & FX & 102 & 409 & 418 & 148 & 3 & MEC & 43.125 & 5 \\
\hline PALIO WK & FIAT & $\mathrm{FX}$ & 108 & 402 & 422 & 152 & 3 & MEC & 45.840 & 5 \\
\hline ASTRA & CHEV & FX & 125 & 332 & 420 & 143 & 3 & MEC & 48.487 & 5 \\
\hline FOCUS & FORD & GAS & 126 & 285 & 415 & 149 & 3 & MEC & 51.365 & 5 \\
\hline MAREA & FIAT & GAS & 132 & 409 & 439 & 143 & 3 & MEC & 54.980 & 5 \\
\hline COROLLA & TOYO & GAS & 136 & 461 & 453 & 148 & 3 & MEC & 57.589 & 5 \\
\hline GOLF & vW & GAS & 116 & 336 & 415 & 146 & 3 & MEC & 58.366 & 5 \\
\hline 307 & PEUG & GAS & 138 & 420 & 420 & 151 & 4 & AUTO & 59.300 & 5 \\
\hline ASTRA SEDAN & CHEV & $\mathrm{FX}$ & 125 & 460 & 434 & 142 & 4 & MEC & 63.198 & 5 \\
\hline VECTRA & CHEV & GAS & 110 & 402 & 450 & 142 & 4 & MEC & 66.851 & 5 \\
\hline A3 & AUDI & GAS & 125 & 303 & 415 & 142 & 4 & AUTO & 75.550 & 5 \\
\hline MAREA WK & FIAT & GAS & 160 & 500 & 449 & 152 & 4 & MEC & 84.190 & 5 \\
\hline STILO & FIAT & GAS & 167 & 380 & 425 & 153 & 4 & MEC & 86.600 & 5 \\
\hline $\mathrm{A} 3$ & AUDI & GAS & 180 & 303 & 415 & 142 & 4 & MEC & 87.124 & 5 \\
\hline
\end{tabular}

Tabela 9: Dados utilizados na simulação de utilização do modelo.

\begin{tabular}{ccccccccccccc}
\hline MOD & MAR & COMB & POT & PM & COMP & ALT & LUX & CAM & PREÇO & NP \\
\hline VECTRA ELEG. & CHEV & GAS & 151 & 526 & 462 & 146 & 5 & AUTO & 84.990 & 5 &
\end{tabular}

LEGENDA:

MOD: Modelo.

REN: Renault.

COM: Combustível.

LUX: Nível de luxo.

CAM: Câmbio.
MAR: Marca. $\quad$ CHEV: Chevrolet.

TOYO: Toyota

GAS: Gasolina.

PM: Porta-malas (I)

MEC: Mecânico.

\section{CITR: Citroën.}

FX: Flex.

ALT: Altura (cm)

AUTO: Automático.
VW: Volkswagen.

PEUG: Peugeot.

POT: Potência (cv)

COMP: Comprimento (cm)

PREÇO: Preço sugerido (RŞ).

NP: $N^{\circ}$ de portas (carros de 2 portas estão como 3 portas e carros de 4 portas como 5 portas). 